\title{
Male Students More Likely to Abuse and Become Addicted to Drugs than Females; A Case Study of Kirinyaga University
}

\author{
Mburu Samuel $^{1}$, Sarah Joy Gitiri ${ }^{2}$, Irene Mutuku ${ }^{3}$ \\ School of Health Sciences, Kirinyaga University, Kenya \\ School of Hospitality, Kirinyaga University, Kenya \\ School of Health Sciences, Kirinyaga University,
}

\begin{abstract}
The use of alcohol and other substances among students at institutions of higher learning impacts negatively on their studies, therefore affecting academic performances. The purpose of this study was to investigate prevalence of alcohol, drugs, other substances use and abuse among students of Kirinyaga University $(K y U)$, categories of drugs used or abused, factors contributing to the use or abuse of the substances, suppliers of the substances, suppliers, impact on performance, memory, extent of addiction to the substances and to propose means of overcoming the challenge. Using a descriptive survey, all registered KyU students (2006) were stratified and systematically sampled using class attendance registers as the sampling frame and programmes as the sampling units. A sample size of $270(n=270)$ was calculated and proportionately allocated to the strata of Degree and Diploma students. Significantly, 34\% of male students' respondents required more of the drugs to get high indicating addiction. Furthermore, there was a significant difference (Chi square value $=3.89 ;$; -value $=0.048) ; P<0.05$ ) between the male and female students' respondents in their requirement for more of the drugs to get high, indicating an increased risk of addiction to the drugs and the other substances. Males student' respondents were more likely to use or abuse drugs, other substances and also more likely to get addicted than their female student' counterparts.
\end{abstract}

Keywords: Alcohol and Drug abuse, Substance abuse in Institutions of higher learning, Youth and drug abuse, Youths and Drug addiction.

\section{INTRODUCTION}

According to World Health Organization (WHO), about 2 billion (33\%) people worldwide consume alcoholic beverages and 76.3 million have diagnosable alcohol use disorders [1]. This renders alcohol the most widely used and abused substance in the world [2]. In addition, the serious health and social effects of alcohol use and abuse makes its prevention and control a public health priority. Importantly, alcohol use and abuse cause 1.8 million deaths annually, a third of which result from unintentional injuries. It also causes a loss of 58.3 million of Disability-Adjusted Life Years (DALY) of which $40 \%$ are due to neuro-psychiatric conditions $[1,3,4$, $5]$.

Alcohol use and abuse is influenced by multiple factors which include gender, family history and parental influence. Although, men are more likely to use and abuse alcohol than women, however the numbers of women drinking, abusing, and becoming addicted to alcohol is rapidly rising [6, 7]. In addition to having problems with job tasks, accidents, and damage to co-worker and customer relations, other short term effects of alcohol use and abuse include loss of work productivity through absenteeism, lateness or leaving early and feeling sick at work $[8,9,10]$. Consequently, this leads to organizational constraints in form of high turn-over and subsequent recruitment, increased consumption of health benefits, in case of illness or accidents that would result to compensation [10]. Furthermore, the psychological pain and suffering experienced by the alcohol users and their significant others and the employer is huge.

According to a countrywide survey by NACADA, (2007) [11], the current alcohol usage defined as consumption in the last 30 days among persons aged 15-65 years was $14.2 \%$, male consumption $22.9 \%$, female consumption being $5.9 \%$, rural consumption $13.0 \%$, urban consumption $17.7 \%$; legal/packaged alcohol $9.1 \%$, traditional liquor 5.5\% and chang'aa $2-3.8 \%$. North Eastern province had the lowest alcohol consumption of $(0$ $\%$ ), followed by Western provinces (6.8\%), while the other six provinces were as follows; Rift Valley $12.5 \%$, Eastern $14.8 \%$, Nyanza $17.0 \%$, Central $17.7 \%$, Coast $18.6 \%$ and Nairobi $-18.6 \%$ ). In addition, more than $80 \%$ of the respondents felt that the second generation alcohol was increasing, while $58 \%$ reported that first generation alcohol was decreasing. Of specific importance, second generation alcohol was found to be the most readily available, affordable and accessible type of alcohol in the provinces, while consumption of alcohol among people aged less than 18 years was rated "high" by significant number of respondents. Worth noting, previous studies have identified idleness; peer pressure; crave for courage or desire to belong to a clique; poor academic performance, lack of role models, ignorance and easy availability of drugs and other substances as the 
main risk factors for alcohol intake [12]. Other factors include unemployment and work or school-related stress. However, the risk factors for alcohol varied by gender. Some of the reasons indicated for taking alcohol include: having fun or to feel good; to relax; to cope with stress; to interact with others and to "kill time" in addition to environmental, biological and psychological factors $[13,14,15]$. Several adverse effects of alcohol abuse to the individual, the household and the community such as episodes of loss of consciousness; inability to meet financial obligations; having multiple sex partners; threatened and attempted suicides; motor vehicle accidents, domestic violence low school enrollment; high school drop-out; poor results in national examinations; decreased employability; marital breakdown; and infertility were identified from the study. In addition, factors such as religious values; parental restrictions; positive peer pressure; work and school commitment; fear of stigmatization and peoples bad experience with alcohol were found to be important protective factors. The most influential factors were identified as the family (erosion of traditional value system and moral decay) [16], peer association, school performance and social class membership. Maithya et al. (2012) [17] and Chesang, (2013) [18] showed a significant relationship between the subjects' drug-using behavior and the involvement of their friends in drugs. The studies indicate that peer pressure encourages youth to use substances under the false impression that some drugs stimulate appetite for food, increase strength and give wisdom as well as courage to face life[16]. Furthermore, the families, especially the parents, are the child's basic socializing agents.] Reports by Maithya, (2012) [17] and Muchemi, (2013) [19] indicated that a child gains his/her first standards of behavior from the parents and other grown-up persons around them. Chesang, (2013) associated delinquency, for example alcohol and marijuana abuse, with lax, inconsistent or abusive parental discipline [16]. Owing to the diverse socialization agents, such as the peer group, teachers, the mass media, the government and the church, and interaction with various people, young people are acquiring values that go beyond those of their immediate localized culture [20].

Across the globe, alcohol and drug abuse is a major challenge to young people [1]. Indeed, use of alcohol and drugs continues to emerge as a strategy for most young people to cope with their prevailing problems such as unemployment, neglect, violence, sexual abuse and poor academic performance [21]. A number of academic research reports and law enforcement reports however, indicate that in the last few years, Kenya has had to deal with an increase in the substance abuse problem. In addition to holding the economy back, drug abuse impacts negatively on young people in learning institutions endangering their lives and the workforce rendering them less productive. Although the actual extent and nature of drug and substance abuse among youth in learning institutions is not clear, research indicates that most students, experiment with and abuse alcohol and other drugs. Studies have indicated that drug and substance abuse was widespread, cutting across all social groups and affecting mostly the youth [22]. According to NACADA, (2012) [20], the majority of students in all levels of education abuse alcohol and other substances and are likely to destroy their lives before they become adults or even drop out of school. This, has been identified as a contributing factor to the current problems experienced in learning institutions in Kenya recently, thus becoming an issue of major concern $[17,22]$.

A report by Chesang, (2013) [18] indicates that by the age of 15, 34\% of this age group had used tobacco, $18 \%$ Cannabis sativa, 32\% had abused miraa (khat) and 5\% cocaine; with the age group at most risk put at between 16 and 18 years. Overall, the most commonly abused drugs were found to be alcohol, tobacco, khat and cannabis. According to national statistics from the Rapid Situation Assessment of Drug and Substance Abuse in Kenya [21], 11.7\% of young people aged 15-24 are current users of alcohol, $6.2 \%$ use tobacco, $4.7 \%$ khat, while $1.5 \%$ are users of Cannabis. In addition, the median age of initiation to tobacco products is 10 years, while the minimum is 8 years. Alarmingly, the median age for alcohol is 10 years and the minimum 4 years. The above statistics indicates dire situation faced by children and the youth in Kenya, who are already burdened by other socio-economic challenges. In addition, availability of imported illegal substances such as heroin, cocaine and mandrax to students has been recognized. However, it was noted that most of the cases alcohol and other substances abuse took place in secondary schools and institutions of higher learning. Importantly, these findings show that drug and substance abuse is a reality among Kenyan youth and there is need for the development and implementation of intervention strategies to curb the problem especially among students [16].

Reports on the impact of substance abuse on academic activities indicate interference with physiological, psychological and emotional functioning [23, 24]. Among the problems recorded are impaired memory and other intellectual faculties, and tracking inability in sensory and perceptual functions [14]. Further, the habit creates preoccupation with acquiring the substances, adverse psycho-pathetic complications, social development leading to truancy and lack of concentration on studies among others [25, 26]. Furthermore, reduced cognitive efficiency leads to poor academic performance and thus resulting in decreased self-esteem. This contributes to instability in an individuals' sense of identity, in turn contributing to further substance abuse and creating a vicious circle $[25,27]$.

Several drug abuse prevention approaches have been proposed; such as selective prevention strategies focusing on helping individuals to develop the knowledge, attitudes and skills they need to make good choices 
or change harmful behaviors. However, lack of policy to guide student leaders on ADA prevention has been identified as the major challenge in addressing drug and substance abuse problems. In addition, some of the students or security officers may collude with drug peddling networks, therefore impeding preventive, elimination strategies and systems. Likewise, lack of privacy during guidance and counseling sessions has been reported to affect negatively the willingness to seek advice by those affected [16], consequently promoting stigmatization.

Maithya et al. (2015) [16], recommended equipping peer counselors and lecturers with counseling skills to effectively deal with drug and substance abusers in learning institutions. In addition, heads of departments and lecturers should give students adequate work in and outside the class throughout the term to reduce idleness, which to some extent contributes to drug and substance abuse. They also suggested strengthening the guidance and counseling departments and ensuring privacy, employment of fulltime counselors in each institution so that students may seek advice. Further, all learning institutions should come up with clear policies on how student leaders should deal with the students who abuse drugs and substances inside the institution. Importantly, an intimate relationship between the dean of students, heads of departments, student leadership and all students should be cultivated to address the problem in a co-operative manner.

Currently, there is no documented data on the prevalence of the use and abuse of the substances among students at KyU. This descriptive survey broad aim of this study was to investigate the prevalence of alcohol, drug and other substance abuse among students of KyU. The investigators were testing the hypothesis that use of alcohol and other substances at KyU among students was widespread. Specifically, the survey investigated the; prevalence and categories of drugs and other substances among students of KyU, suppliers of the substances, impact on functioning, performance, memory, level of dependence, factors contributing to the use, abuse of the substances and propose means of overcoming the challenge.

\section{METHODS}

Design

This was a descriptive survey conducted at KyU whereby, self-administered questionnaires were distributed, filled after a signed informed consent had been obtained from every participant. KyU is one of the recently chartered public Universities in Kenya. The University is located approximately 115 Km North East of Nairobi off Nairobi Sagana-Embu-Highway in Kutus Town, the headquarters of Kirinyaga County, in the former Central province of Kenya. The questionnaires were numbered, coded and information transcribed into SPSS software (IBM version 23), analyzed by descriptive statistics and interpreted.

Sample population

The population comprised randomly generated sample of students both male and females. Inclusion and Exclusion criteria

All KyU registered students at the time of sampling, which included all registered degree, diploma students (who appeared in the class attendance registers) as at the time of the study (2006), were included. All unregistered students (not in the class attendance registers) as at the time of the survey were excluded from the study.

Sampling Frame

The class attendance registers was used as the sampling frame to generate a systematic random sample for the students of KyU respectively.

Sampling Unit

The departments and academic programmes were used as the sampling units for random sampling of students of KyU respectively.

Sampling

Probability sampling strategy of proportional stratified random sampling followed by a systematic random sampling (selecting samples at equal intervals) was employed in this survey to avoid selection bias, for external validity (generalizability) and to control for confounding effect at design stage of the descriptive survey. The sample population was stratified into two strata, sub-strata of degree and diploma students and males and females (during analysis).

Sample size computation

In the calculation of sample size, the following variables were applied: Design effect of 2, Correlation variation (CV) of 5\%, P of 5\% and adjustment for non-response of 5\%. A total sample size of $270(\mathrm{n}=270)$ was gotten and proportionately allocated and distributed to participants strata according to the ratio of degree students and diploma students from a total population of 2006 registered students.

Sampling

The questionnaires were pre-tested before the survey to know the time taken to fill them and review answerability of the questions. The questionnaires with an attached consent cover letter were distributed to the participants in the specific survey strata for self-administration. A systematic random sampling of selecting 
samples at specific equal intervals using sampling frames (class attendance registers) of the different strata was employed.

Data collection, analysis and interpretation

After return of the filed questionnaires, they were numbered according to strata, the data/response coded and data transcribed into SPSS (IBM version 23) and analyzed using descriptive statistics. During analysis stage, the data was stratified in two; students, males and females to control for confounding effect. The results were analyzed and reported as percentages, summary table and bar graphs. A $p=\leq 0.05$ was used to judge the significance.

\section{RESULTS}

The results of this descriptive survey were summarized and presented in form of a summary table (as shown in Table 1)

Table I: The Impact of Drugs, Other Substances on Students Respondents' Functioning, Performance, Memory and Dependence.

\begin{tabular}{|c|c|c|c|c|c|c|}
\hline \multirow[t]{2}{*}{ Impact of Drug Use on Students } & \multicolumn{3}{|c|}{ Male } & \multicolumn{3}{|c|}{ Female } \\
\hline & $\mathrm{N}$ & $\mathrm{n}$ & $\%$ & $\mathrm{~N}$ & $\mathrm{n}$ & $\%$ \\
\hline Uses drugs occasionally & 39 & 11 & (28) & 30 & 6 & (20) \\
\hline Requires more to get high* & 38 & 13 & (34) & 30 & 4 & (13) \\
\hline Functions best when high & 39 & 11 & (28) & 30 & 4 & (13) \\
\hline Wanted to reduce intake & 38 & 4 & (11) & 25 & 5 & (20) \\
\hline Failed to do ones duties & 37 & 7 & (19) & 25 & 5 & (20) \\
\hline Unable to remember things & 37 & 10 & (27) & 25 & 5 & (20) \\
\hline Tried to cut down but couldn't & 37 & 6 & (16) & 26 & 4 & (15) \\
\hline Sometimes unable to control your consumption & 37 & 10 & (27) & 26 & 6 & (23) \\
\hline
\end{tabular}

Table 1 shows the impact of drugs and other students on functioning, performance, intake, memory, addiction. The table shows $34 \%$ of males students requiring more of the drugs to get high an indication of an addiction, memory of $27 \%$ of the male students had been impaired by the drugs and $27 \%$ of the male addicted to drugs and unable to control their consumption. Notably, $20 \%$ of females used drugs occasionally could not be able to reduce their intake, failed to perform their duties due to drugs, their memory had been impaired by drugs. Significantly, $27 \%$ of males and $23 \%$ of the female students were addicted to the drugs and unable to control their consumption $(*$ Chi square value $=3.89$; $\mathrm{p}$-value $=0.048$ ); $\mathrm{P}<0.05$. There was a significant difference between the male and female students' population in their requiring more of the drugs to get high, indicating an increased risk of addiction to the drugs and the other substances.

\section{DISCUSSION}

This aim of this descriptive survey was to investigate the prevalence of alcohol, drug and other substance abuse among students of KyU. The investigators were testing the hypothesis that use of alcohol and substance abuse among KyU students was widespread. Specifically the survey investigated the; prevalence and categories of drugs and other substances among students of KyU, suppliers of the substances, impact on functioning, performance, memory, level of dependence, factors contributing to the use, abuse of the substances and propose means of overcoming the challenge. The study population comprised of randomly sampled KyU students. In this survey, majority of the student's respondents were between 16-30 years of age.

Significantly, in six of the leading drugs and other substances used or abused at KyU (i.e. wine, beer, miraa/khat, spirits, Cannabis sativa\& hashish), male users were notably higher than females, which was in agreement with studies by Chesang et al. (2013)[18] and Maithya et al. (2015) [16] as mentioned in the introduction. In addition, $27 \%$ of male respondents were addicted to drugs, other substances, thus unable to control their consumption of drugs and other substances compared to only $23 \%$ of female' respondents. Likewise, according to Table 1,34\% of male student respondents required more of the drugs to get high, notably indicating addiction, which was in support of the study by Agrawal \& Dick (2008)[14]. Importantly, there was a significant difference ( $*$ Chi square value $=3.89$; $\mathrm{p}$-value $=0.048) ; \mathrm{P}<0.05$ ) between the male and female students' respondents in their requirement for more of the drugs to get high, indicating an increased risk of addiction to the drugs and the other substances. Accordingly, males student' respondents were more likely to use or abuse drugs, other substances and are also more likely to get addicted than their female student' counterparts.

Furthermore, the results indicated that cigarettes, khat, Cannabis, beer, spirits and smokeless tobacco were the mostly supplied substances at $\mathrm{KyU}$ which, in addition to wine, cigar, hashish also happens to be the most frequently used or abused substances. Subsequently, this finding supports that of Maithya et al. (2015) 
[16] in their study on effects of drugs and other substances on academic participation among Technical institute students in Kenya. According to the survey, $25 \%$ of the male and $19 \%$ of the female students used drugs occasionally, of which beer, wine, spirits, Cannabis and khat were the most used or abused in that order. This was in agreement with a previous survey by Chesang, (2013) [18]. Importantly, majority of the students were introduced to the substances by friends or their relatives supporting the findings by Chesang et al. (2013) [18] and Maithya et al. (2012) [17] as mentioned previously in the introduction.

Although the main source of money for the substances for the student respondents was either parents or self, it is worth noting that a good number of them reported betting through SportsPesa as their source of income. Accordingly, majority of the study respondents spent between 500-3000 Kenya shillings on the substances monthly. The emerging of the often addictive culture of betting or gambling among the students should sound warning bells to management of the institutions due to the snowballing effect of this vice. Another important red flag from the survey was the indication of fellow students, community members, gang members, other sources such as pubs and surrounding shops as their main suppliers of the drugs. This indicated that mechanisms of surveillance within the institution and its environs was inadequate and required strengthening.

Of concern, but in agreement with Eisenstein, (2005) [25] and Agrawal \& Dick, (2008) [14], the impact of drugs, others substances on the performance, functioning, memory, dependence on the student respondents was evident as indicated by the $25 \%$ of the male, $13 \%$ of the female respondents, who reported functioning best when high, $16 \%$ of the male students, $15 \%$ of female students who reported addiction by having tried to cut down on consumption, $27 \%$ of males, $23 \%$ females student respondents addicted to drugs, other substances or unable to control their consumption at all (as shown in Table I). In addition, 34\% of the male students reported requiring high volumes of alcohol to get high, with $24 \%$ of males indicating to function better when high. Interestingly, $20 \%$ of the females and $11 \%$ female student reported wanting to reduce their intake of alcohol. Significantly, Table I shows $20 \%$ of the females and $19 \%$ of the male students who were unable to perform their duties due to alcohol intake in addition to $27 \%$ males, $20 \%$ female students whose memory had been impaired to an extent they were unable to remember things due to alcohol intake. This was in agreement with a report by Chermacket al. (2008) [28].

Despite several measures taken by the government to curb alcohol and drug abuse such as the formation of NACADA, the practice is still prevalent in institutions of higher learning. This means that alcohol, drug and other substances abuse among students in Kenya has become a serious problem that affects all parts of the Country [16]. Recently, the use and abuse of second generation, illicit brew and other alcoholic drinks by the youth in Kenya, specifically in Central province had reached a crisis level [21]. Consequently, this prompted the president to declare war on manufacturers, retailers and consumers of illicit brews and in particular second generation wines and spirits. Specifically, institutions of higher learning have not been spared either. Hence, the use or abuse of alcohol, drugs, other substances has become rampant leading to serious adverse effects on health and performance of students of KyU. Availability of extra finances, from the higher loans education board (HELB), County and national government bursary funds, emerging sports betting culture coupled by lack of financial management skills, negative peer pressure, social media, information explosion, advanced technology have complicated alcohol and drug abuse (ADA) prevention strategies.

As a way forward, management of institutions of higher learning should put mechanisms for surveillance within or surrounding environs, identifying, dealing with suppliers of the substances from the neighboring community and fellow students. Further, they should increase support, welfare systems for students, sensitization and awareness efforts (e.g. ADA prevention committees, developing and operationalizing workrelated ADA prevention policies). Innovative, creative methodologies to make learning interesting, mentally stimulating, student mentorship programmes, teaching life skills, entrepreneurship and financial management skills to students should be encouraged. Likewise, developing critical, creating thinking capacities for students to make independent decisions to withstand, navigate successfully throughthe negative influences from peer pressure, available technology and information explosion will be important. Moreover, other suggestions include; keeping students engaged during weekends, after classes, enhancing guidance, counseling, involving student unions leadership, hostel managers, surrounding communities by training, sensitization and awareness campaigns on ADA prevention. Additionally, training on ethics, values, all-round development, discovering one's life purpose, integrity, effects of sports betting, gambling in general, awareness, sensitization campaigns, peer counseling, increase of recreation extra-curricular facilities, academic mentorship, career guidance, counseling, extra-curricular activities, academic discussion, support groups, increased academic assignments are promising strategies to fight drug and other substances use and abuse at institutions of higher learning [29]. Collaboration with provincial administration (local chiefs, County coordinators to regulate sports betting) involving "Nyumba Kumi", security committees, local police, County government security, youth and County affair services in case of local brews and local drug suppliers, religious organizations should be explored to prevent and stop the vice before it spills out control. 


\section{CONCLUSION}

In conclusion, according to the results, male students were more likely to abuse drugs than their female counterparts. Therefore, preventive and corrective measures need to be taken. The most commonly used and abused substances were wine, beer, khat, spirits, Cannabis sativa and hashish. From the survey, negative effects of the use, abuse of drugs and other substances at institutions of higher learning were evident. This was based on the number of respondents who failed to perform their duties, could only function best when high, unable to remember things, unable to cut down on use or their consumption and therefore addicted. The main suppliers of these substances were fellow students, community members and gang members. Of major concern and requiring immediate action was the emerging addictive culture of sports betting or gambling as a source of income among the student respondents. This calls for preventive and interventional strategies to nip the vice in the bud.

\section{CONFLICT OF INTEREST}

The authors would like to declare that there was no conflict of interest in conducting and publication of this descriptive survey.

[1]. World Health Organization. Global Status Report on Alcohol. Geneva, 2004. http://www.who.int/substance_abuse/publications/global_status_report_2004_overview.pdf. Downloaded on $18^{\text {th }}$ November, 2016.

[2]. D. Basangwa et al. "Alcohol and substance and mental disorders." Tropical Medicine and International Health, 15, 2006, 34.

[3]. World Health Organization. (2002). Alcohol in Developing Societies: A Public Health Approach. Geneva. http://apps.who.int/iris/handle/10665/42623. Downloaded on 20 ${ }^{\text {th }}$ November, 2016.

[4]. W.M Compton et al. "Prevalence, Correlates, Disability and Comorbidity of DSM-IV Drug Abuse and Dependence in the United States." Archives of General Psychiatry, 64, 5, 2007, 566-576.

[5]. World Health Organization. (2014). Global Status Report on Alcohol. Geneva. http://www.who.int/substance_abuse/publications/global_alcohol_report/msb_gsr_2014_1.pdf. Downloaded on $20^{\text {th }}$ January 2017.

[6]. S. Schinke \& T. Schwinn. "Gender-Specific Computer-Based Intervention for Preventing Drug Abuse among Girls." American Journal of Drug and Alcohol Abuse, 31, 2005, 609-616.

[7]. T. Flensborg-Madsen et al. Amount of alcohol consumption and risk of developing alcoholism in men and women. Alcohol and Alcoholism,42(5), 2007, 442.

[8]. T.C Blum et al. Alcohol Consumption and Work Performance. Journal of Studies on Alcohol, 54, 1993, $61-70$

[9]. E. Gordis. Alcohol and the Workplace. A Commentary by the Director of National Institute of Alcohol Abuse and Alcoholism (NIAAA) 1999.

[10]. NACADA (National Authority for the Campaign against Alcohol and Drug Abuse 2007). Rapid situation assessment of drug and substance abuse in Kenya. Nairobi: NACADA.

[11]. T.Korhonen. "Physical Activity in Adolescence as a Predictor of Alcohol and Illicit Drug Use in Early Adulthood: A Longitudinal Population-Based Twin Study." Twin Research in Human Genetics, 12, 2009, 261-268.

[12]. J. Randerson. Alcohol Worse Than Ecstasy on Shock New Drug List, (2007). http://www.maps.org/media/view/alcohol_worse_than_ecstasy_on_shock_new_drug_list/. Downloaded on $20^{\text {th }}$ February, 2017.

[13]. D.M Dick \& A. Agrawal. The genetics of alcohol and other drug dependence. Alcohol Research \& Health, 31(2), 2008, 111-119.

[14]. S.Kuppin, R.M Carpiano. Public Conceptions of Serious Mental Illness and Substance Abuse, Their Causes and Treatments: Findings from the 1996 General Social Survey". American Journal of Public Health, 96(10), 2006, 1766-1771.

[15]. National Campaign against Drug Abuse Authority. (2012). Rapid Assessment of Drug and Substance Abuse in Kenya. https://scadkenya.files.wordpress.com/2017/01/rapid-assessment-drug-and-substanceabuse-situation-kenya-20122.pdf. Downloaded on $10^{\text {th }}$ January, 2017

[16]. D.Emmite\& S.J Swierzewski. Risk Factors and causes of Alcohol Abuse. 2008, http://www.mentalhealthchannel.net/alcohol/riskfactors.shtml, Downloaded on $20^{\text {th }}$ January, 2017.

[17]. R.Maithya et al. A Baseline Survey on Effects of Drug and Substance Abuse on Academic Participation among Students in Technical Institutions in Kenya. International Journal of Education and Research, 3, $2015,1$.

[18]. R.K Chesang. Drug Abuse among the youth in Kenya. International Journal of Scientific and Technology Research, 2(6), 2013, 126-131. 
[19]. R.Muchemi. Drug and substance abuse among youth in the informal settlements: A Paper presented at NACADA Conference, 2013.

[20]. R.M Etheridge et al. "Drug Abuse Treatment and Comprehensive Services for Adolescents." Journal of Adolescent Research, 16, 2001, 563-589.

[21]. J.D Hawkins, R.F Catalano \& JY Miller. Risk and protective factors for alcohol and other drug problems in adolescence and early adulthood: implications for substance abuse prevention. Psychological bulletin, 112(1), 1992, 64.

[22]. T.K Eisenstein, RT Rahim, P Feng, N K Thingalaya, \& J JMeissler. Effects of opioid tolerance and withdrawal on the immune system. Journal of Neuroimmune Pharmacology, 1(3), 2006, 237-249.

[23]. National Campaign against Drug Abuse Authority (NACADA). Fact Finding Mission. Report on the Extent of Alcohol and Drug Abuse in Central Province. 2009.

[24]. F.Ducci et al. "HTR3B Is Associated With Alcoholism with Antisocial Behavior and Alpha EEG-Power - An Intermediate Phenotype for Alcoholism and Co-Morbid Behaviors." Alcohol, 43, 2009, 73-84.

[25]. M. T Fillmore. "Drug Abuse as a Problem of Impaired Control: Current Approaches and Findings." Behavioral Cognitive Neuroscience Review, 2, 2003, 179.

[26]. S.T. Chermack et al. "Partner Aggression among Men and Women in Substance Use Disorder Treatment: Correlates of Psychological and Physical Aggression and Injury." Drug and Alcohol Dependence, 98, 2008, 35-44.

[27]. R.E Drake \& K.T Mueser. "Psychosocial Approaches to Dual Diagnosis." Schizophrenia Bulletin, 26,2000, 105-118.

[28]. R.E Drake \& M.A Wallach. "Dual Diagnosis: 15 Years of Progress."Psychiatric Services, 51, 2000, 1126-1129.

[29]. S. Farrer. "School-Based Program Promotes Positive Behavior, Reduces Risk Factors for Drug Use, Other Problems." National Institute of Drug Abuse, 18, 2004, 6. 